\title{
Radio stars for linking celestial reference frames
}

\author{
H.G. Walter ${ }^{1}$, R. Hering ${ }^{1}$, and Chr. de Vegt ${ }^{2}$ \\ 1 Astronomisches Rechen-Institut, Mönchhofstrasse 12-14, D-69120 Heidelberg, Germany \\ 2 Hamburger Sternwarte, Gojenbergsweg 112, D-21029 Hamburg, Germany
}

Received August 12; accepted August 22,1996

\begin{abstract}
Radio stars play a key role in establishing the link between optical reference frames and the conventional celestial reference frame based on extragalactic radio sources. The relevant astrometric, astrophysical and radio quantities are compiled of 66 cardinal radio stars currently suited to frame connection and maintenance of the link. The catalogue entries are supplied with ample bibliographical codes and annotations for easy data retrieval. ${ }^{1}$
\end{abstract}

Key words: catalogues — astrometry - reference frames - radio continuum: stars

\section{Introduction}

Radio stars are suitable objects for an immediate connection of stellar reference frames to radio reference frames and vice versa. From the positions of the stars in both frames follows the orientation of one frame with respect to the other at some specified epoch; the spin is zeroized by means of the proper motions. At present, in view of the limited number of radio stars the determination of the orientation and spin parameters is best performed by rigid rotations. This mode of execution is even more suggested when it can be taken for granted that the stars representing the frames constitute rigid spheres.

A practical application of this method is the determination of the link of the provisional Hipparcos frame to the extragalactic reference frame based on radio sources (Lindegren \& Kovalevsky 1995). This frame has been established by IERS (IERS 1994) and is going to be adopted by IAU as the conventional celestial reference frame (ICRF).

The IAU Joint Working Group on Reference Frames (IAU 1992, 1996) at its meeting in Hamburg, 1994 March

Send offprint requests to: $\mathrm{R}$. Hering

1 The catalogue is available electronically at the CDS via anonymous ftp 130.79.128.5 and via http://cdsweb.u-strasbg.fr/Abstract.html
28-29, has tentatively selected some 50 radio stars as candidates for setting up the link to the ICRF and for maintaining the link. Clearly, this explains the important role of radio stars not only for the presence but also in future. It is therefore not surprising that a growing body of opinion among the astronomical community called for a revision of the existing data on radio stars.

Since radio stars are found among a large variety of classes of stars such as single stars, binaries, variable stars, masers etc., the stellar data are widely scattered, a fact which suggests a consistent data assembling and the compilation of a catalogue of radio stars. In contrast to the optical data, an extensive collection of radio properties has been made by Wendker (1995). The search of radio positions of high precision depends almost exclusively on the VLBI, VLA and MERLIN networks.

Our catalogue contains updated astrometric and astrophysical data of the radio stars chosen by the working group in 1994 augmented by a dozen of stars that have become eligible for the link in the meantime. Further, we give keys that allow the retrieval of detailed radio information and the identification of the interferometry networks, which have the individual star on their observing programme. All in all the catalogue comprises 66 objects. Because of their status they may be called "the cardinal radio stars for linking celestial frames". Their sky distribution is plotted in Fig. 1. The region below $-30^{0}$ of declination is missing among the stars recommended by the Working Group since the radio stars confirmed in this part of the sky are not yet sufficiently explored by radio interferometry.

At least for some time the observing efforts will concentrate on the cardinal radio stars aiming at the determination of precise radio proper motions for the entire set. To extend the set of cardinal radio stars one may take advantage of a fund of confirmed and suspected radio stars for astrometry which are listed in a former catalogue by Walter et al. (1990) including 186 objects most of them being Hipparcos stars. The majority of the cardinal radio stars are among these objects, but many stars of the former catalogue are either suspected radio stars due to a low 
flux density or their radio positions are derived from single dish observations thus leaving unresolved radio/optical identification problems.

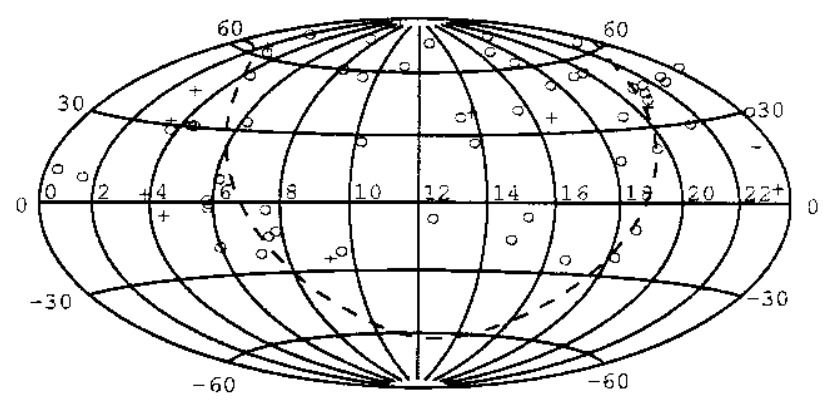

Fig. 1. Cardinal radio stars for the link of optical reference frames to the extragalactic reference frame. The sky distribution is shown in equatorial coordinates, the dotted line marks the galactic equator. Circles and crosses refer to stars with optical positions and, apart from a few exceptions, with positions from radio interferometry. More specifically, crosses indicate stars with VLBI positions

In Sect. 2 the potential of radio stars for connecting frames is briefly exemplified by the results obtained from preliminary Hipparcos catalogue versions. Section 3 describes the organization of the catalogue which finally follows in the annex.

\section{Use of radio stars for the Hipparcos link}

A preliminary link of the Hipparcos frame to the 3 axes of the ICRF has been established by means of 7 stars whose positions and proper motions are known from VLBI observations on the one hand and from the reduction of the 30-months Hipparcos data by the FAST consortium on the other (Kovalevsky et al. 1995). According to Lestrade et al. (1995), the rotation angles of frame orientation at epoch 1991.25 are in units of 1 milliarcsecond (mas)

$A_{1}=-26.9 \pm 0.5, A_{2}=-12.6 \pm 0.6, A_{3}=23.0 \pm 0.5$,

and the rotation rates giving the relative spin are in units of $1 \mathrm{mas} / \mathrm{yr}$

$\dot{A}_{1}=0.6 \pm 0.5, \dot{A}_{2}=0.1 \pm 0.5, \dot{A}_{3}=1.4 \pm 0.4$.

On the analogy of VLBI we undertook a pilot study using 10 stars with VLA positions (Florkowski et al. 1985) and Hipparcos positions from 37 months of data of the FAST consortium (Lindegren \& Kovalevsky 1995). The 10 VLA link stars were selected by applying a $2 \sigma$ rejection criterion to the post-fit residuals HIP-VLA; their designations are: b Per, HD 50896, KQ Pup, FK Com, HR 5110, $\beta$ Lyr, BD +43 3571, RT Lac, AR Lac and HD 224085.

Since VLA based radio proper motions were not available, only the position link could be established. Using
Hipparcos proper motions in place of the radio proper motions for unavoidable epoch transformations, the following rotation angles of the frame orientation in units of mas at epoch 1992.0 were obtained:

$A_{1}=-13.6 \pm 11.2, A_{2}=-22.4 \pm 11.6, A_{3}=13.7 \pm 11.7$.

Note that the quoted rotation angles and precisions result from pilot studies on the Hipparcos link and are presented here to illustrate the capability of VLA observations. The official link of the Hipparcos frame to the extragalactic frame will be provided by ESA.

Due to the lower precision of the VLA positions, i.e. 30 mas as compared with roughly 1 mas for VLBI, it is not surprising that the VLA results on the rotation angles are inferior to VLBI. Nevertheless, the signs and magnitudes of the rotation angles show the same tendency for the VLBI and VLA observations. From extrapolating the VLA results a precision of 5 mas is estimated for the rotation angles when some 50 objects are involved in the leastsquares adjustment process. In the course of the application of the high sensitive VLBA technique (Kellermann \& Thompson 1985) an increase of number and positional accuracy of radio stars is expected and, thus, radio stars will continue to play also in future the role of intermediaries in optical and radio astrometry.

\section{Conception of the catalogue}

\subsection{The catalogue structure}

The catalogue is intended to aid all those who engaged in linking of reference frames by means of radio stars, and in the maintenance of the links. Accordingly, it also addresses observers who provide in future observations of stars at optical and radio wavelengths. To this end we structured the catalogue by categories that are divided into three main groups of data: astrometric quantities including double and multiple stars, astrophysical quantities, and ancillary quantities giving references to finding charts, radio maps, radio emission and interferometer networks.

With regard to the star designation we tried to cover a wide range of names to simplify the identification of objects. As far as the other categories are concerned it was our aim to make primarily one single entry that reflects the optimum quantity of this category. Clearly, the choice was not always unambiguous, a fact which led occasionally to several entries. Since only those data appear in the catalogue that are published in the literature and have received a quotation, the term "optimum" should be seen in this context. Especially the astrometric entries suffer from the restriction to quotable data, because many of them are still in the pipeline and their publication is tied to the finalization of the respective projects, such as the Hipparcos data reduction or campaigns of radio interferometry. To enable the conscientious catalogue user of taking avantage 
of data whose publication is imminent, we have given ample star identifications in the name list, for instance the Hipparcos star number, and references to the interferometer networks dealing with one or other of the stars. Hence, cross-referencing to any sort of data is made easy. In addition, the bibliographical codes associated with each entry facilitate the deepening of data retrieval.

Transformations of positions and proper motions from B1950.0 to J2000.0 were performed along the lines of Aoki et al. (1983) and Schwan (1988). The catalogue is an extract from a comprehensive data base of radio stars maintained at the Astronomisches Rechen-Institut, which in addition keeps on file a machine-readable catalogue version.

\subsection{The catalogue formats}

Per star a maximum of 18 categories of stellar parameters were entered in the catalogue, each category consisting of one or more records and accompanying references. Throughout the catalogue a uniform system of units was applied. The detailed definition of the categories and adopted units will be found in the catalogue header.

Category 1: Star designations are listed in 3 columns of 20 characters each per entry. If available, the following six designations are arranged in hierarchical order: (1) A 4-digit working number precedes the customary designations to permit easy cross-referencing to the reservoir of radio stars by Walter et al. (1990), (2) Durchmusterungsnumber (BD, CoD, CPD), (3) HD number, (4) PPM number, (5) Hipparcos Input Catalogue number (HIC), (6) variable star designation; (7) and so on reserved for other designations in alphabetical order.

Category 2: Optical position: Right ascension (RA) and standard deviation, declination (Dec) and standard deviation, referred to J2000.0 at observation epoch; observation epochs in RA and Dec; the reference system of positions (usually the FK5 system). Normally the parallax effect is removed from the coordinates. For certainty, consultation of the reference is suggested.

Category 3: Radio positions: RA and standard deviation, Dec and standard deviation, referred to J2000.0 at observation epoch; observation epochs in RA and Dec; the reference system of the calibrators. Concerning the parallax effect see Category 2.

Category 4: Optical proper motions in RA and Dec referred to J2000.0; the reference system of proper motions (usually the FK5 system).

Category 5: Radio proper motions in RA and Dec referred to J2000.0; the reference system of the calibrators.

Category 6: Optical parallax.

Category 7: Radio parallax.

Category 8: Radial velocities.
Category 9: Apparent magnitude of the star and the respective photometric system $(U, B, V, R, P, H$ (Hipparcos), $K$ (Kukarkin)). For variable stars normally maximum and minimum values of magnitude, and the period are quoted. For double and multiple systems, occasionally, the integrated magnitude is given supplemented by the magnitudes of the components.

Category 10: Type of variability.

Category 11: Spectral type and luminosity class of star or system. If available, the respective quantities of the components are listed too.

Category 12: Optical structure.

Category 13: Properties of double stars: Type of binary or multiple system, number of components, separation of components, position angles, magnitudes.

Category 14: Guide to finding charts.

Category 15: Guide to radio maps.

Category 16: Guide to radio emission.

Category 17: Observation priority, membership to observing programmes.

Category 18: Miscellaneous (X-ray source, emission line star, star association, etc.).

Whenever a category is void it has been omitted from the records of the respective star.

The bibliographical codes accompanying the entries are compiled at the end of the catalogue under the heading "References". The list of references is arranged both in order of ascending bibliographical codes and in alphabetical order of the authors' names.

\subsection{The catalogue data}

For exemplification we present in Table 1 the sample of the entries of star $1028=\mathrm{BD}+280532=\mathrm{HD} 21242=$ PPM $92507=\mathrm{HIC}$ 16042. The catalogue as a whole is available at the $\mathrm{CDS}$.

\section{Conclusions}

In this catalogue the stellar data and keys to data retrieval are supplied of the presently 66 cardinal radio stars that have the potential of connecting optical and radio celestial reference frames. The catalogue is thought to be an aid of studies on linking frames, and of repeated observations of the radio stars of this core in view of the maintenance of the link. A survey of supplementary radio star candidates is urged for enlarging the core in order to obtain better uniformity of the spatial distribution, the distribution of spectral types and apparent magnitudes. More diversification of the types of radio stars will diminish the risk of biases. 
Table 1. Catalogue entries of star $1028=$ BD $+280532=$ HD $21242=$ PPM $92507=$ HIC 16042

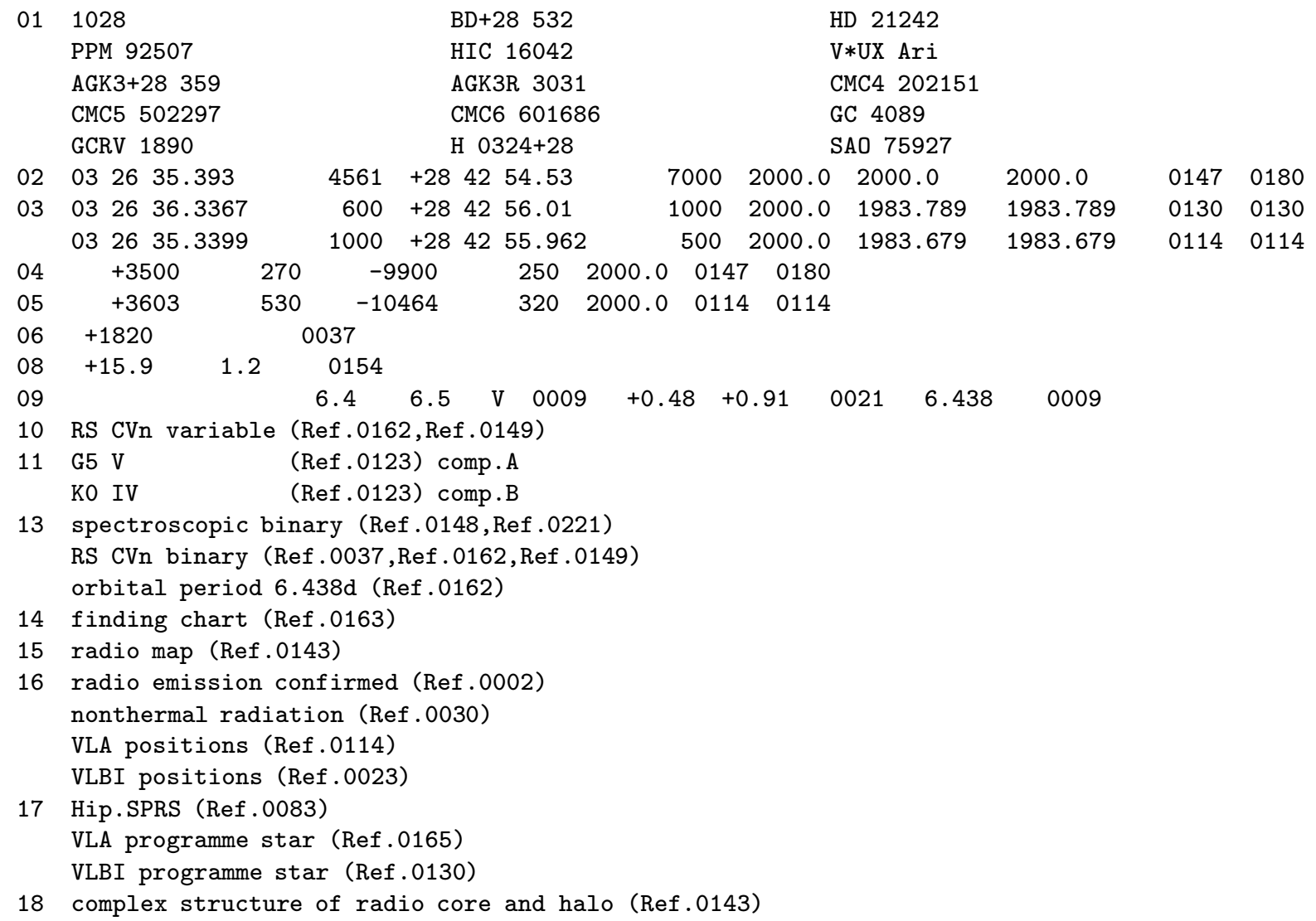

Acknowledgements. This research has made use of the SIMBAD data base, operated at CDS, Strasbourg, France. C.d.V. wants to thank the Bundesministerium für Bildung, Wissenschaft, Forschung und Technologie (BMBF) for financial support under grant No. 50008810 (Hipparcos).

\section{References}

Aoki S., Sóma M., Kinoshita H., Inoue K., 1983, A\&A 128, 263 Florkowski D.R., Johnston K.J., Wade C.M., de Vegt C., 1985, AJ 90, 2381

IAU, 1992, Inform. Bull. 67, 33

IAU, 1996, Transactions of the International Astronomical
Union, Vol. XXII B. In: Appenzeller I. (ed.). Kluwer Acad. Publ., Dordrecht Boston London, p. 140

IERS, 1994, Implementation of the IERS Celestial Reference Frame. In: 1993 IERS Annual Report. Observatoire de Paris, p. II-23

Kellermann K.I., Thompson A.R., 1985, Sci 229, 123

Kovalevsky J., Lindegren L., Froeschlé M. et al., 1995, A\&A 304,34

Lestrade J.-F., Jones D.L., Preston R.A. et al., 1995, A\&A 304, 182

Lindegren L., Kovalevsky J., 1995, A\&A 304, 189

Schwan H., 1988, A\&A 198, 363

Walter H.G., Hering R., de Vegt Chr., 1990, A\&AS 86, 357

Wendker H.J., 1995, A\&AS 109, 177 